\title{
EVOLUTION OF COGNITIVE DEMAND IN THE HUMAN-MACHINE INTERACTION INTEGRATED WITH INDUSTRY 4.0 TECHNOLOGIES
}

\author{
MARIANNA MADONNA ${ }^{1}$, LUIGI MONICA $^{2}$, SARA ANASTASI $^{2} \&$ MARIO DI NARDO $^{3}$ \\ ${ }^{1}$ INAIL UOT CVR, Italy \\ ${ }^{2}$ INAIL DIT, Italy \\ ${ }^{3}$ Department of Materials Engineering and Operations Management, Faculty of Engineering, \\ University of Naples "Federico II", Italy
}

\begin{abstract}
In the highly technological and innovative scenario of Industry 4.0, characterized by a series of enabling technologies and a strong interconnection of resources, it is necessary to take into account the impact that the introduction of increasingly sophisticated sensors and collaborative machines on the safety aspects. In addition to the introduction of so-called "smart" technologies, the context of the use of new technologies and the tasks of front-line operators has also changed. The worker increasingly assumes the role of supervisor and when some types of work require particular human skills, there is a real "collaboration" between man and machine. In the new factories, the interaction with "smart machines" on one hand simplifies the operations of the worker making them less complex and less susceptible to errors and on the other hand increases the information and communication of these systems and leads to a complexity that requires new man-machine interface modes. The support of sensors and new technologies allows the detection of a series of data necessary to make the most reliable predictions on the state of health of the equipment so that it is possible to plan target interventions. This implies that the cognitive interaction effort of the machine operator moves from the skill level to the knowledge level because the human is required to manage a huge amount of data (big data) that must be acquired, analysed and interpreted. This paper, starting from consolidated human reliability methodologies in the literature, which allows for evaluating human error in different work fields, aims to highlight how human performance improves even if it implies an increase in cognitive demand due to the use of new smart technologies.
\end{abstract}

Keywords: Industry 4.0, smart technologies, human-machine interaction, human factors and behaviour.

\section{INTRODUCTION}

The new connectivity and the interaction technologies among the elements of the sociotechnical system, which characterized Industry 4.0, modify the traditional relationship manmachine-environment. The Internet of Things (IoT) allows the organizations to gather vast amount of data from smart devices equipped on processes, machines and products so that they allow monitoring their status and taking actions in real-time. The enabling technologies, such as virtual and augmented reality, collaborative robots, exoskeletons, support and aid human work. However, the advanced technologies reduce operator's physical workload, also allowing humans to perform their job more safely, but they require new demands of operators' skills and competencies. In fact, the control of intelligent machines requires a new cognitive load as the lower skilled human jobs were replaced by advanced technology while many high precision and complex processes are still managed by humans or by robots collaboration thanks to advanced human-machine interface (HMI) [1].

In this new scenario, the key factor for 4.0 competitiveness of companies will be the presence of highly qualified personnel, able to guarantee not only the implementation of new technologies but also the correct supervision of plant processes by the latter. 
The paper aims, in the first section, to describe the new role of the front-line operator in the smart factories and in the second section to define a conceptual cognitive framework of operator 4.0.

\section{EVOLUTION OF THE ROLE OF FRONT-LINE OPERATOR OVER TIME}

According to Romero et al. [2], the role of the operator in production has changed following the industrial revolutions that have taken place over the time, as shown in Fig. 1.

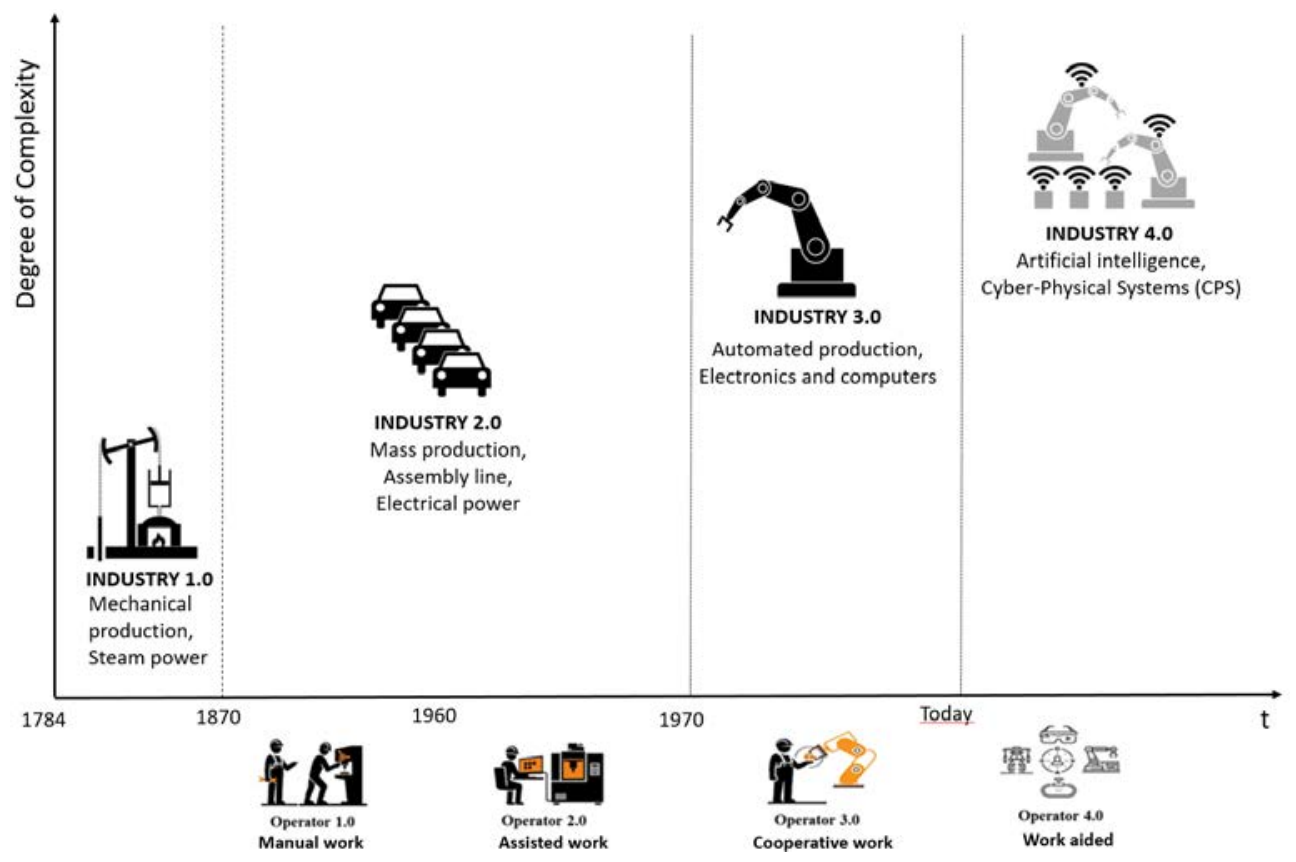

Figure 1: Operator evolution according to industrial revolutions. (Adapted from Romero et al. [2].)

Each industrial revolution is characterized by an increasing degree of complexity of the technologies involved and by a different role of the front-line operator who works with them. The Operator 1.0 generation is defined in [2] as "humans conducting manual and dextrous work with the support from mechanical tools and manually operated machine tools". The Operator 2.0 performs "assisted work" with the support of computer tools (e.g. Computer Numerical Control) while the Operator 3.0 performs "cooperative work" with robots and other machine and computer tools, also known as human robot collaboration. The Operator 4.0 is "the operator of the future", a smart and skilled operator whose physical, sensory and cognitive capabilities are enhanced using advanced enabling technologies.

\subsection{The new role of operator in smart factory}

As the smart factory is characterized by an increasingly need for flexibility and adaptability of production systems, an increased cognitive workload of the operator 4.0 is required (e.g. diagnosis, situation awareness, decision-making, planning, etc.). 
According to the vision of the operator 4.0 in Romero et al. [3], that depicts how the enabling technologies in the Industry 4.0 enhance the operator in carry out the task, eight types of operators are identified; they interact physically and cognitively with the technology:

1. Super-Strength Operator who uses exoskeleton;

2. Collaborative Operator who uses a collaborative robot;

3. Virtual Operator who uses virtual reality;

4. Augmented Operator who uses augmented reality;

5. Smarter Operator who uses intelligent personal assistant;

6. Social Operator who uses social networks;

7. Analytical Operator who uses Big Data analytics;

8. Healthy Operator who uses a wearable tracker [4].

In this paper, focused on human-machine interface, only cognitive interactions are considered:

Smarter Operator: an operator helped by an intelligent personal assistant (software agent or artificial intelligence) in performing his tasks handsfree by means of voice interaction technology. This interaction has positive repercussions on the safety of the operator as it reduces the error probability especially in searching and retrieving from a digital library through voice commands. Many advantages are also in maintenance activities when the instructions are read to the operator while he performs tasks.

Virtual Operator: an operator aided by an advanced simulation of realistic environment so that some activities are carried out in safer manner. For example, virtual reality can help operator to perform tasks, by reducing error probability as it improves operator training on new assembly and maintenance procedures.

Augmented Operator: an operator whose field of view of the real factory environment is enriched, by means of smartphones, tablet, etc., with digital information. This technology offers many advantages in terms of reducing human errors as it supports operator in carrying out his task by becoming digital assistance and improving decision-making when feedbacks are displayed in real-time.

Analytic Operator: this operator uses big data analytics, the process of collecting, organizing and analyzing amount of data in order to gather useful information to predict events. The increasing available data by means of cheap sensors and Internet of Things allow the operator to receive in real time warnings about machine fault and to make predictions more accurately [4].

\section{THE EVOLUTION OF COGNITIVE DEMAND}

The human reliability analysis (HRA) methods have been developed to quantify human error probability in performing an assigned task, so they needed to formalize a model of human behaviour that describes the cognitive process of human beings and its link with human performance [5].

The aim of this paper is to show how human performance improves even if it implies an increase in cognitive demand due to the use of new smart technologies, therefore there is the need to take as reference a cognitive model.

Therefore the model considered is that underlying the Cognitive Reliability and Error Analysis Method (CREAM), one of the second generation of HRA techniques [6].

Earlier versions of the CREAM made use of a simplified model of cognition called SMoC, which just means Simple Model of Cognition (Hollnagel and Cacciabue [7]).

The purpose of SMoC was to describe the human cognition based on four basic cognitive functions: observation, interpretation, planning and execution. The main feature of the SMoC 
is the cyclical nature of human cognition, as it implied atypical path from observation over interpretation and planning to execution. It can generate any sequential model, including the well-known step-ladder cognitive model (Rasmussen [8]), which is structured on three different pathways of increasing complexity that require increasing levels of attention and cognitive resources.

According to mental model described in CREAM, cognition should not be described as a sequence of steps, but as a controlled use of the available competence (skills, procedures, and knowledge) and resources. So that, each typical cognitive activity can be described in terms of combination of the four cognitive functions it requires.

The cognitive profile is based on a table of the cognitive functions associated with each of the cognitive activity as shown below in Table 1, based on a Simple Model of Cognition. The following list of cognitive activities derived from several source based on assumption that they are exhaustive in pragmatic sense but from analytical point of view it is impossible to prove that they are complete, consistent, or even correct.

Table 1: A generic cognitive activity by cognitive demand matrix. (Adapted from [6].)

\begin{tabular}{|c|c|c|c|c|}
\hline \multirow{2}{*}{ Activity type } & \multicolumn{4}{|c|}{ Cognitive function } \\
\hline & Observation & Interpretation & Planning & Execution \\
\hline Co-ordinate & & & $\mathbf{X}$ & $\mathbf{X}$ \\
\hline Communicate & & & & $\mathbf{X}$ \\
\hline Compare & & $\mathbf{X}$ & & \\
\hline Diagnose & & $\mathbf{X}$ & $\mathbf{X}$ & \\
\hline Evaluate & & $\mathbf{X}$ & $\mathbf{X}$ & \\
\hline \multicolumn{5}{|l|}{ Execute } \\
\hline Identify & & $\mathbf{X}$ & & \\
\hline Maintain & & & $\mathbf{X}$ & $\mathbf{X}$ \\
\hline Monitor & $\mathbf{X}$ & $\mathbf{X}$ & & \\
\hline Observe & $\mathbf{X}$ & & & \\
\hline Plan & & & $\mathbf{X}$ & \\
\hline Record & & $\mathbf{X}$ & & $\mathbf{X}$ \\
\hline Regulate & $\mathbf{X}$ & & & $\mathbf{X}$ \\
\hline Scan & $\mathbf{X}$ & & & \\
\hline Verify & $\mathbf{X}$ & $\mathbf{X}$ & & \\
\hline
\end{tabular}

As the first CREAM step, we want to build a profile of the cognitive demands of one of type of human-enabling technology interaction as characterized by Romero et al. [3]. In particular, in order to put into practice the construction of the cognitive profile, an operator intervention by means of augmented reality in a maintenance activity [9], is considered.

3.1 The cognitive demand in human-enabling technology interaction in maintenance activity: an example

An operator in a shop floor monitors the health status of the machines and the process parameters via display. At some point, an alarm is displayed on his control station. The alarm refers to a fault on a machine that forms paper envelopes. Since the machine is equipped with 
sensors, they transmit signals on its status to operator's workstation. On the display, the machine scheme is shown in its entirety and the fault is precisely located. The area of the machine affected by the fault that led to the machine down, is highlighted in red colour, precisely the area between the unfolding of the paper roll and the entrance of it into the rollers. The operator is trained to intervene with the support of augmented reality. The display of his workstation is that of a portable tablet. He removes it from the fixed base and moves himself in front of the machine where the fault occurred. The operator selects the augmented reality mode on the tablet. In this way, as augmented reality has been applied to all the machines in the shop-floor, when the operator photographs the machine, a library opens up display where it is possible to select an interactive use and maintenance manual. Furthermore, by placing the tablet's camera towards the machine, a series of additional information are displayed such as danger and warning signs relating to the dangerous areas of the machine and the PPE (personal protective equipment) necessary to operate on it. The operator puts the suggested PPE and takes the labelled toolbox to operate on that machine.

The operator selects from the library, which has a set of on-line tools [10], the use and maintenance manual and in particular the procedure for restoring the functionality in that area of the machine affected by the paper jam.

The selected procedure is explained step by step and simultaneously shown on the display with the appropriate danger warnings. The operator adheres to the procedure in the execution and completes it successfully and safely.

In Table 2, the maintenance activity, described above, has been divided into elementary tasks. The relative cognitive activity was associated to each task and therefore, through Table 1 , the cognitive functions involved are identified.

Table 2: Cognitive demands table for maintenance activity aided with augmented reality.

\begin{tabular}{|l|l|l|l|l|l|}
\hline Task step or activity & $\begin{array}{c}\text { Cognitive } \\
\text { activity }\end{array}$ & Obs & Int & Plan & Exe \\
\hline Keep track of machine state over the time & Monitor & $\mathrm{x}$ & $\mathrm{x}$ & & \\
\hline Establish the identity of machine state & Identify & & $\mathrm{x}$ & & \\
\hline $\begin{array}{l}\text { Assess the actual situation based on available } \\
\text { information }\end{array}$ & Evaluate & & $\mathrm{x}$ & $\mathrm{x}$ & \\
\hline $\begin{array}{l}\text { Organise a set of actions by which the repair } \\
\text { will be successfully achieved }\end{array}$ & Plan & & & $\mathrm{x}$ & \\
\hline $\begin{array}{l}\text { Perform specific actions according to step by } \\
\text { step procedures displayed on tablet }\end{array}$ & Execute & & & & $\mathrm{x}$ \\
\hline \begin{tabular}{l} 
Check the feedback from prior operations \\
\hline
\end{tabular}
\end{tabular}


As result, the cognitive demand in this maintenance activity aided with augmented reality, implies all four cognitive functions, with prevalence of the interpretation function, followed by observation and planning and finally by execution. Therefore, it is possible to deduce that the use of advanced technologies of Industry 4.0 requires a considerable cognitive commitment. However, it is worth highlighting how the use of augmented reality offers the operator a real-time training with step-by-step procedures such as to minimize errors due to the incorrect or omitted execution of one of the elementary tasks.

\section{A CONCEPTUAL FRAMEWORK OF THE OPERATOR 4.0 COGNITIVE MODEL}

In Fig. 2, the cognitive model of operator in smart factory is schematized in a conceptual framework. A huge quantity of data is collected from physical world by means of sensors on equipment, processes and products. By means of IoT, data are transmitted and displayed on the operator interface technologies. In this way, the operator become a data receiver of the Cyber-Physical System (CPS), the virtual twin of physical system, keeping the process parameters and the health status of the machines under control. The data collected and displayed on the human technology interface are processed, first implying the cognitive functions of observation and interpretation and after requiring knowledge-based activities that involve the planning and the execution of the consequent actions. The new humanmachine interaction implies all four cognitive functions and the advanced technologies enhance operator to perform tasks more reliably. In fact, the data related to process status are more reliable (so the observation and interpretation processes are less susceptible to human error) and the augmented and virtual reality assist the operator in the process of decision making (planning) and in acting (execution) in a safer way thanks to procedures dictated step by step.

\section{CONCLUSION}

The new cognitive demand of front-line operator is due to the increasing complexity of the information displayed, the need to interpret the content of such information and, if necessary,

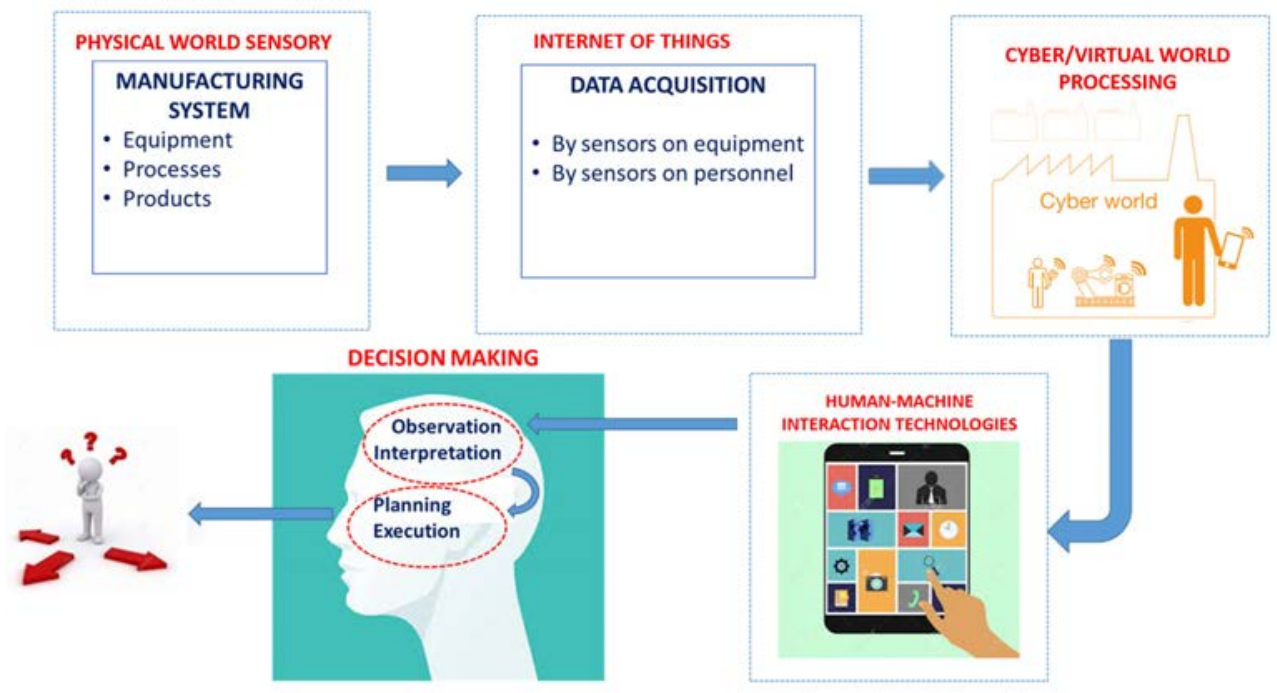

Figure 2: A conceptual cognitive framework of operator in smart factory. 
to promptly act. For this reason, the HMI plays an important role, as it has to guide and teach the user in a virtual or augmented environment. As worker's competence profile changes, teaching and training are necessary in updating the skills required.

In the future, it would be interesting to compare the probability of human error of an operator who works in a traditional way with that of an operator using enabling Industry 4.0 technologies. The assessment of the human probability error (HPE) could be performed using one of the human reliability techniques available in literature. In accordance with the analysis conducted in this paper, the CREAM is well suited for this purpose.

Furthermore, another aspect to consider is that the virtual and the augmented reality could be useful tools in training, as they allow the operator to act in dynamic and unforeseen situation safely also by means of on-line step-by-step procedures [11].

In this highly innovative scenario, the human resource requires more competences and skills to manage new technologies. Therefore, the challenge that companies will have to face will be knowledge management that becomes another strategic innovation factor.

\section{REFERENCES}

[1] Peruzzini, M., Grandi, F. \& Pellicciari, M., Exploring the potential of Operator 4.0 interface and monitoring. Computer and Industrial Engineering, 2018.

[2] Romero, D., Bernus, P., Noran, O., Stahre, J. \& Fast-Berlung, Å. The Operator 4.0: Human cyber-physical systems and adaptive automation towards human-automation symbiosis work system. IFIP International Conference on Advances in Production Management Systems, pp. 677-686, 2016.

[3] Romero, D. et al., Towards an Operator 4.0 typology: A human-centric perspective on the fourth industrial revolution technologies. International Conference on Computers and Industrial Engineering (CIE46), pp. 1-11, 2016.

[4] Zolotovà, I., Papcun, P., Ajàti, E., Miskuf, M. \& Mocnej, J., Smart and cognitive solutions for Operator 4.0: Laboratory H-CPPS case study. Computer and Industrial Engineering, 2018.

[5] Di Nardo, M., Gallo, M., Madonna, M. \& Santillo, L.C. A conceptual model of human behaviour in socio-technical systems. Communications in Computer and Information Science, 532, pp. 598-609, 2015.

[6] Hollnagel, E., Cognitive Reliability and Error Analysis Method: CREAM, Elsevier, 1998.

[7] Hollnagel, E. \& Cacciabue, C., Modeling cognition and erroneous actions in system simulation contexts. The 3rd European Meeting on Cognitive Science Approaches to Process Control, 1991.

[8] Rasmussen, J., Information Processing and Human-Machine Interaction: An Approach to Cognitive Engineering, Wiley, 1986.

[9] Bottani, E. \& Vignali, G., Augmented reality technology in the manufacturing industry: A review of the last decade. IISE Transactions, 51(3), pp. 284-310, 2019.

[10] Lotti, G., Villani, V., Battilani, N. \& Fantuzzi, C., Towards an integrated approach for supporting the workers in Industry 4.0. Proceedings of IEEE Industrial CyberPhysical Systems, ICPS, 2018.

[11] Villani, V., Sabattini, L., Czerniak, J.N., Mertens, A., Vogel-Heuser, B. \& Fantuzzi, C., Towards modern inclusive factories: A methodology for the development of smart adaptive human-machine interfaces. Proceedings IEEE International Conference on Emerging Technologies and Factory Automation, ETFA, 2017. 